International Journal of Pure and Applied Mathematics

Volume 86 No. 5 2013, 833-847

ISSN: 1311-8080 (printed version); ISSN: 1314-3395 (on-line version)

url: http://www.ijpam.eu

doi: http://dx.doi.org/10.12732/ijpam.v86i5.7

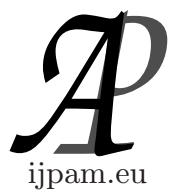

\title{
RIGHT AND LEFT ROUGH SETS INDUCED BY FUNCTIONS
}

\author{
Yong Chan Kim \\ Department of Mathematics \\ Gangneung-Wonju University \\ Gangneung, Gangwondo, 210-702, KOREA
}

\begin{abstract}
In this paper, we investigate the properties of right and left rough sets induced by functions on a generalized residuated lattice. In particular, we construct right (left) closure (interior) operators.
\end{abstract}

AMS Subject Classification: 03E72, 54A40, 54B10

Key Words: complete residuated lattice, right and left rough sets, right (left) closure (interior) operators

\section{Introduction}

Pawlak $[8,9]$ introduced the rough set as a mathematical tool for dealing information as the uncertainty of data in engineering, physics, computer sciences and many other diverse field. Recently, Bělohlávek [1-3] investigate the properties of fuzzy rough set on a residuated lattice which supports part of foundation of theoretic computer science. Chen and Li [3] introduced fuzzy rough sets induced by functions. Georgescu and Popescue [4.5] introduced non-commutative fuzzy Galois connection in a generalized residuated lattice which is induced by two implications.

In this paper, we investigate the properties of right and left rough sets induced by functions on a generalized residuated lattice. In particular, we construct right (left) closure (interior) operators.

Received: May 9, 2013

(c) 2013 Academic Publications, Ltd. url: www.acadpubl.eu 


\section{Preliminaries}

Definition 1. (see [4,5]) A structure $(L, \vee, \wedge, \odot, \rightarrow, \Rightarrow, \perp, \top)$ is called a generalized residuated lattice if it satisfies the following conditions:

(GR1) $(L, \vee, \wedge, \top, \perp)$ is a bounded lattice where 1 is the universal upper bound and 0 denotes the universal lower bound;

(GR2) $(L, \odot, \top)$ is a monoid;

(GR3) it satisfies a residuation, i.e.

$$
a \odot b \leq c \text { iff } a \leq b \rightarrow c \text { iff } b \leq a \Rightarrow c .
$$

We call that a generalized residuated lattice has the law of double negation if $a=\left(a^{*}\right)^{0}=\left(a^{0}\right)^{*}$ where $a^{0}=a \rightarrow \perp$ and $a^{*}=a \Rightarrow \perp$.

Remark 2. (see [1-7]) (1) A generalized residuated lattice is a residuated lattice $(\rightarrow=\Rightarrow)$ iff $\odot$ is commutative.

(2) A left-continuous t-norm $([0,1], \leq, \odot)$ defined by $a \rightarrow b=\bigvee\{c \mid a \odot c \leq$ $b\}$ is a residuated lattice $\mathrm{xms}$

(3) A pseudo MV-algebra is a generalized residuated lattice with the law of double negation.

In this paper, we assume $(L, \wedge, \vee, \odot, \rightarrow, \Rightarrow, \perp, \top)$ is a complete generalized residuated lattice with the law of double negation.

Lemma 3. (see $[4,5]$ ) For each $x, y, z, x_{i}, y_{i} \in L$, we have the following properties.

(1) If $y \leq z,(x \odot y) \leq(x \odot z), x \rightarrow y \leq x \rightarrow z$ and $z \rightarrow x \leq y \rightarrow x$ for $\rightarrow \in\{\rightarrow, \Rightarrow\}$. Xms

(2) $x \odot y \leq x \wedge y \leq x \vee y$.

(3) $x \rightarrow\left(\bigwedge_{i \in \Gamma} y_{i}\right)=\bigwedge_{i \in \Gamma}\left(x \rightarrow y_{i}\right)$ and $\left(\bigvee_{i \in \Gamma} x_{i}\right) \rightarrow y=\bigwedge_{i \in \Gamma}\left(x_{i} \rightarrow y\right)$ for $\rightarrow \in\{\rightarrow, \Rightarrow\}$.

(4) $x \rightarrow\left(\bigvee_{i \in \Gamma} y_{i}\right) \geq \bigvee_{i \in \Gamma}\left(x \rightarrow y_{i}\right)$, for $\rightarrow \in\{\rightarrow, \Rightarrow\}$.

(5) $\left(\bigwedge_{i \in \Gamma} x_{i}\right) \rightarrow y \geq \bigvee_{i \in \Gamma}\left(x_{i} \rightarrow y\right)$, for $\rightarrow \in\{\rightarrow, \Rightarrow\}$.

(6) $(x \odot y) \rightarrow z=x \rightarrow(y \rightarrow z)$ and $(x \odot y) \Rightarrow z=y \Rightarrow(x \Rightarrow z)$.

(7) $x \rightarrow(y \Rightarrow z)=y \Rightarrow(x \rightarrow z)$ and $x \Rightarrow(y \rightarrow z)=y \rightarrow(x \Rightarrow z)$.

(8) $x \odot(x \rightarrow y) \leq y$ and $(x \Rightarrow y) \odot x \leq y$.

(9) $(x \Rightarrow y) \odot(y \Rightarrow z) \leq x \Rightarrow z$ and $(y \rightarrow z) \odot(x \rightarrow y) \leq x \rightarrow z$. 
(10) $(x \Rightarrow z) \leq(y \odot x) \Rightarrow(y \odot z)$ and $(x \rightarrow z) \leq(x \odot y) \rightarrow(z \odot y)$.

(11) $(x \Rightarrow y) \leq(y \Rightarrow z) \rightarrow(x \Rightarrow z)$ and $(y \Rightarrow z) \leq(x \Rightarrow y) \Rightarrow(x \Rightarrow z) \mathrm{xms}$

(12) $x_{i} \rightarrow y_{i} \leq\left(\bigwedge_{i \in \Gamma} x_{i}\right) \rightarrow\left(\bigwedge_{i \in \Gamma} y_{i}\right)$ for $\rightarrow \in\{\rightarrow, \Rightarrow\}$.

(13) $x_{i} \rightarrow y_{i} \leq\left(\bigvee_{i \in \Gamma} x_{i}\right) \rightarrow\left(\bigvee_{i \in \Gamma} y_{i}\right)$ for $\rightarrow \in\{\rightarrow, \Rightarrow\}$.

(14) $x \rightarrow y=\top$ iff $x \leq y$.

(15) $x \rightarrow y=y^{0} \Rightarrow x^{0}$ and $x \Rightarrow y=y^{*} \rightarrow x^{*}$.

(16) $\bigwedge_{i \in \Gamma} x_{i}^{*}=\left(\bigvee_{i \in \Gamma} x_{i}\right)^{*}$ and $\bigvee_{i \in \Gamma} x_{i}^{*}=\left(\bigwedge_{i \in \Gamma} x_{i}\right)^{*}$.

(17) $\bigwedge_{i \in \Gamma} x_{i}^{0}=\left(\bigvee_{i \in \Gamma} x_{i}\right)^{0}$ and $\bigvee_{i \in \Gamma} x_{i}^{0}=\left(\bigwedge_{i \in \Gamma} x_{i}\right)^{0}$

Definition 4. Let $X$ be a set. A function $e_{X}^{r}: X \times X \rightarrow L$ is called:

(E1) $e_{X}^{r}(x, x)=\top$ for all $x \in X$,

(E2) If $e_{X}^{r}(x, y)=\top$ and $e_{X}^{r}(y, x)=\top$, then $x=y$,

(R) $e_{X}^{r}(x, y) \odot e_{X}^{r}(y, z) \leq e_{X}^{r}(x, z)$, for all $x, y, z \in X$.

Then $e_{X}^{r}$ is called a right partial order. If $e_{X}^{l}$ satisfies (E1), (E2) and

(L) $e_{X}^{l}(y, z) \odot e_{X}^{l}(x, y) \leq e_{X}^{l}(x, z)$, for all $x, y, z \in X$.

Then $e_{X}^{l}$ is called a left partial order. The triple $\left(X, e_{X}^{r}, e_{X}^{l}\right)$ is called a bi-partial ordered set.

Example 5. (1) We define two functions $e_{L}^{r}, e_{L}^{l}: L \times L \rightarrow L$ as $e_{L}^{r}(x, y)=$ $x \Rightarrow y$ and $e_{L}^{l}(x, y)=x \rightarrow y$. Then $e_{L}^{r}$ is a right partial order and $e_{L}^{l}$ is a left partial order.

(2) We define two functions $e_{L^{X}}^{r}, e_{L^{X}}^{l}: L^{X} \times L^{X} \rightarrow L$ as

$$
e_{L^{X}}^{r}(A, B)=\bigwedge_{x \in X}(A(x) \Rightarrow B(x)), e_{L^{X}}^{l}(A, B)=\bigwedge_{x \in X}(A(x) \rightarrow B(x)) .
$$

By Lemma 3(9), $e_{L^{X}}^{r}$ is a right partial order and $e_{L^{X}}^{l}$ is a left partial order.

Definition 6. An operator $I^{r}: L^{X} \rightarrow L^{X}$ is called a right interior operator on $X$ if it satisfies the following conditions:

(I1) $I^{r}(A) \leq A, \mathrm{xms}$

(I2) $I^{r}\left(I^{r}(A)\right) \geq I^{r}(A)$,

(RI) $e_{L^{X}}^{r}(A, B) \leq e_{L^{X}}^{r}\left(I^{r}(A), I^{r}(B)\right)$.

The pair $\left(X, I^{r}\right)$ is called a right interior space.

An operator $I^{l}: L^{X} \rightarrow L^{X}$ is called a left interior operator on $X$ if it satisfies the conditions (I1),(I2), and 
(LI) $e_{L^{X}}^{l}(A, B) \leq e_{L^{X}}^{l}\left(I^{l}(A), I^{l}(B)\right)$.

An operator $C^{r}: L^{X} \rightarrow L^{X}$ is called a right closure operator on $X$ if it satisfies the following conditions:

(C1) $C^{r}(A) \geq A$,

(C2) $C^{r}\left(C^{r}(A)\right) \leq C^{r}(A)$,

$(\mathrm{RC}) e_{L^{X}}^{r}(A, B) \leq e_{L^{X}}^{r}\left(C^{r}(A), C^{r}(B)\right)$.

The pair $\left(X, C^{r}\right)$ is called an $r$-closure space.

An operator $C^{l}: L^{X} \rightarrow L^{X}$ is called a left closure operator on $X$ if it satisfies the conditions $(\mathrm{C} 1),(\mathrm{C} 2)$ and

(RC) $e_{L^{X}}^{l}(A, B) \leq e_{L^{X}}^{l}\left(C^{l}(A), C^{l}(B)\right)$.

Definition 7. Let $\left(X, e_{X}^{r}, e_{X}^{l}\right)$ be a bi-partial ordered set and $A \in L^{X}$.

(1) A point $x_{0}$ is called a right join of $A$, denoted by $x_{0}=\sqcup_{r} A$, if it satisfies

(RJ1) $A(x) \leq e_{X}^{r}\left(x, x_{0}\right)$,

(RJ2) $\bigwedge_{x \in X}\left(A(x) \Rightarrow e_{X}^{r}(x, y) \leq e_{X}^{r}\left(x_{0}, y\right)\right.$.

(2) A point $x_{0}$ is called a left join of $A$, denoted by $x_{0}=\sqcup_{l} A$, if it satisfies

(LJ1) $A(x) \leq e_{X}^{l}\left(x, x_{0}\right)$,

$(\mathrm{LJ} 2) \bigwedge_{x \in X}\left(A(x) \rightarrow e_{X}^{l}(x, y) \leq e_{X}^{l}\left(x_{0}, y\right)\right.$.

An bi-partial ordered set $\left(X, e_{X}^{r}, e_{X}^{l}\right)$ is called a right (resp. left) join complete lattice if $\sqcup_{r} A$ ( resp $\sqcup_{r} A$ ) exists for $A \in L^{X}$.

Remark 8. Let $\left(X, e_{X}^{r}, e_{X}^{l}\right)$ be a bi-partial ordered set and $A \in L^{X}$. If $x_{0}$ is a right join of $A$, then it is unique because $e_{X}^{r}\left(x_{0}, y\right)=e_{X}^{r}\left(y_{0}, y\right)$ for all $y \in X$, put $y=x_{0}$ or $y=y_{0}$, then $e_{X}^{r}\left(x_{0}, y_{0}\right)=e_{X}^{r}\left(y_{0}, x_{0}\right)=\top$ implies $x_{0}=y_{0}$. Similarly, if left join of $A$ exist, then it is unique.

Theorem 9. Let $\left(X, e_{X}^{r}, e_{X}^{l}\right)$ be a bi-partial ordered set and $A \in L^{X}$.

(1) $x_{0}$ is a right join of $A$ iff $\bigwedge_{x \in X}\left(A(x) \Rightarrow e_{X}^{r}(x, y)\right)=e_{X}^{r}\left(x_{0}, y\right)$.

(2) $x_{0}$ is a left join of $A$ iff $\bigwedge_{x \in X}\left(A(x) \rightarrow e_{X}^{l}(x, y)=e_{X}^{l}\left(x_{0}, y\right)\right.$.

Proof. (1) $(\Rightarrow)$ Let $x_{0}$ be a right join of $A$. Then $A(x) \leq e_{X}^{r}\left(x, x_{0}\right)$. Thus, $A(x) \odot e_{X}^{r}\left(x_{0}, y\right) \leq e_{X}^{r}\left(x, x_{0}\right) \odot e_{X}^{r}\left(x_{0}, y\right) \leq e_{X}^{r}(x, y)$. Hence $e_{X}^{r}\left(x_{0}, y\right) \leq$ $\bigwedge_{x \in X}\left(A(x) \Rightarrow e_{X}^{r}(x, y)\right)$. By (RJ2), the equality holds.

$(\Leftarrow)$ Since $\bigwedge_{x \in X}\left(A(x) \Rightarrow e_{X}^{r}\left(x, x_{0}\right)\right)=e_{X}^{r}\left(x_{0}, x_{0}\right)=1$, then $A(x) \leq$ $e_{X}^{r}\left(x, x_{0}\right)$. Hence the result holds. 
Remark 10. Let $\left(L^{X}, e_{L^{X}}^{r}, e_{L^{X}}^{l}\right)$ be a bi-partial ordered set and $A \in L^{L}$.

(1) Since $\sqcup_{r} \Phi$ is a right join of $\Phi$ iff

$$
\bigwedge_{A \in L^{X}}\left(\Phi(A) \Rightarrow e_{L^{X}}^{r}(A, B)\right)=e_{L^{X}}^{r}\left(\bigvee_{A \in L^{X}}(A \odot \Phi(A), B)=e_{L^{X}}^{r}\left(\sqcup_{r} \Phi, B\right),\right.
$$

then $\sqcup_{r} \Phi=\bigvee_{A \in L^{X}}(A \odot \Phi(A))$.

(2) Since $\sqcup_{l} \Phi$ is a left join of $\Phi$ iff

$$
\bigwedge_{A \in L^{X}}\left(\Phi(A) \rightarrow e_{L^{X}}^{l}(A, B)\right)=e_{L^{X}}^{l}\left(\bigvee_{A \in L^{X}}(\Phi(A) \odot A), B\right)=e_{L^{X}}^{l}\left(\sqcup_{l} \Phi, B\right),
$$

then $\sqcup_{l} \Phi=\bigvee_{A \in L^{X}}(\Phi(A) \odot A)$.

\section{Right and Left Rough Sets Induced by Functions}

Definition 11. Let $\left(L^{X}, e_{L^{X}}^{r}, e_{L^{X}}^{l}\right)$ be a bi-partial ordered set and $M=$ $\{\{a / x\} \mid a \in L, a \neq \perp, x \in X\}$ be the set of all singletons. Let $\phi: M \rightarrow L^{X}$

$$
\begin{gathered}
\rho_{r}(A, B)=\bigvee_{x \in X}(A(x) \odot B(x)), \rho_{l}(A, B)=\bigvee_{x \in X}(B(x) \odot A(x)) . \\
N_{r}(A)(x)=\bigvee_{\{a / x\} \in M}\left(a \odot e_{L^{X}}^{r}(\phi(\{a / x\}), A)\right) . \\
N_{l}(A)(x)=\bigvee_{\{a / x\} \in M}\left(e_{L^{X}}^{l}(\phi(\{a / x\}), A) \odot a\right) . \\
H_{r}(A)(x)=\bigvee_{\{a / x\} \in M}\left(a \odot \rho_{r}(\phi(\{a / x\}), A)\right) . \\
N_{l}(A)(x)=\bigvee_{\{a / x\} \in M}\left(\rho_{l}(\phi(\{a / x\}), A) \odot a\right) .
\end{gathered}
$$

If $N_{r}(A)=H_{r}(A)$ (resp. $\left.N_{l}(A)=H_{l}(A)\right)$, then $A$ is a right (resp. left) definable set. The pair $\left(N_{r}(A), H_{r}(A)\right)$ (resp. $\left.\left(N_{l}(A), H_{l}(A)\right)\right)$ is called a right (resp. left) rough set. 
Theorem 12. (1) $N_{r}\left(\top_{X}\right)=\top_{X}$ and $N_{l}\left(\top_{X}\right)=\top_{X}$ where $\top_{X}(x)=\top$ for all $x \in X$.

(2) $H_{r}\left(\perp_{X}\right)=\perp_{X}$ and $H_{l}\left(\perp_{X}\right)=\perp_{X}$ where $\perp_{X}(x)=\perp$ for all $x \in X$.

(3) $\rho_{r}(A, B) \odot e_{L^{X}}^{r}(B, C) \leq \rho_{r}(A, C)$ and $e_{L^{X}}^{l}(B, C) \odot \rho_{l}(A, B) \leq \rho_{l}(A, C)$.

(4) $e_{L^{X}}^{r}(A, B) \leq e_{L^{X}}^{r}\left(N_{r}(A), N_{r}(B)\right)$ and $e_{L^{X}}^{l}(A, B) \leq e_{L^{X}}^{l}\left(N_{l}(A), N_{l}(B)\right)$.

(5) $e_{L^{X}}^{r}(A, B) \leq e_{L^{X}}^{r}\left(H_{r}(A), H_{r}(B)\right)$ and $e_{L^{X}}^{l}(A, B) \leq e_{L^{X}}^{l}\left(H_{l}(A), H_{l}(B)\right)$.

Proof. (1) and (2)

$$
\begin{aligned}
N_{r}\left(\top_{X}\right)(x) & =\bigvee_{\{a / x\} \in M}\left(a \odot e_{L^{X}}^{r}\left(\phi(\{a / x\}), \top_{X}\right)\right) \\
& =\bigvee_{\{a / x\} \in M}(a \odot \top)=\top . \\
H_{r}\left(\perp_{X}\right)(x) & =\bigvee_{\{a / x\} \in M}\left(a \odot \rho_{r}\left(\phi(\{a / x\}), \perp_{X}\right)\right) \\
& =\bigvee_{\{a / x\} \in M}(a \odot \perp)=\perp .
\end{aligned}
$$

Other cases are simiarly proved.

(3)

$$
\begin{aligned}
\rho_{r}(A, B) \odot e_{L^{X}}^{r}(B, C) & =\bigvee_{x \in X}(A(x) \odot B(x)) \odot \bigwedge_{y \in Y}(B(y) \Rightarrow C(y)) \\
& \leq \bigvee_{x \in X}(A(x) \odot B(x)) \odot(B(x) \Rightarrow C(x)) \\
& \leq \bigvee_{x \in X}(A(x) \odot C(x))
\end{aligned}
$$

$$
\begin{aligned}
N_{r}(A)(x) \odot e_{L^{X}}^{r}(A, B) & =\bigvee_{\{a / x\} \in M}\left(a \odot e_{L^{X}}^{r}(\phi(\{a / x\}), A)\right) \odot e_{L^{X}}^{r}(A, B) \\
& \leq \bigvee_{\{a / x\} \in M}\left(a \odot e_{L^{X}}^{r}(\phi(\{a / x\}), B)\right) \\
& \leq N_{r}(B)(x) .
\end{aligned}
$$

So, $e_{L^{X}}^{r}(A, B) \leq N_{r}(A)(x) \Rightarrow N_{r}(B)(x)$.

$$
\begin{aligned}
e_{L^{X}}^{l}(A, B) \odot N_{l}(A)(x) & =e_{L^{X}}^{l}(A, B) \odot \bigvee_{\{a / x\} \in M}\left(e_{L^{X}}^{l}(\phi(\{a / x\}), A) \odot a\right) \\
& =\bigvee_{\{a / x\} \in M}\left(\left(e_{L^{X}}^{l}(A, B) \odot e_{L^{X}}^{l}(\phi(\{a / x\}), A)\right) \odot a\right) \\
& \left.=\bigvee_{\{a / x\} \in M}\left(e_{L^{X}}^{l}(\phi(\{a / x\}), B)\right) \odot a\right) \\
& \leq N_{l}(B)(x) .
\end{aligned}
$$

So, $e_{L^{X}}^{l}(A, B) \leq N_{l}(A)(x) \rightarrow N_{l}(B)(x)$. 
(5)

$$
\begin{aligned}
& H_{r}(A)(x) \odot e_{L^{X}}^{r}(A, B) \\
& =\bigvee_{\{a / x\} \in M}\left(a \odot \rho_{r}(\phi(\{a / x\}), A)\right) \odot e_{L^{X}}^{r}(A, B) \\
& \leq \bigvee_{\{a / x\} \in M}\left(a \odot \bigvee_{y \in X}(\phi(\{a / x\})(y) \odot A(y)) \odot(A(y) \Rightarrow B(y))\right) \\
& \leq \bigvee_{\{a / x\} \in M}\left(a \odot \bigvee_{y \in X}(\phi(\{a / x\})(y) \odot B(y))\right) \\
& =H_{r}(B)(x)
\end{aligned}
$$

So, $e_{L^{X}}^{r}(A, B) \leq H_{r}(A)(x) \Rightarrow H_{r}(B)(x)$.

$$
\begin{aligned}
& e_{L^{X}}^{l}(A, B) \odot H_{l}(A)(x) \\
& =e_{L^{X}}^{l}(A, B) \odot \bigvee_{\{a / x\} \in M}\left(\rho_{l}(\phi(\{a / x\}), A) \odot a\right) \\
& \left.\left.=\bigvee_{\{a / x\} \in M} \bigvee_{y \in X}((A(y) \rightarrow B(y)) \odot A(y) \odot \phi(\{a / x\})(y))\right) \odot a\right) \\
& \left.=\bigvee_{\{a / x\} \in M}\left(\rho_{l}(\phi(\{a / x\}), B)\right) \odot a\right) \\
& \leq H_{l}(B)(x) .
\end{aligned}
$$

So, $e_{L^{X}}^{l}(A, B) \leq H_{l}(A)(x) \rightarrow H_{l}(B)(x)$.

Theorem 13. Let $\phi(\{a / x\})=\{a / x\}$. Then

(1) $N_{r}(A)=A$ and $N_{l}(A)=A$.

(2) $A \leq H_{r}(A)$ and $A \leq H_{l}(A)$.

Proof. (1)

$$
\begin{aligned}
& N_{r}(A)(x)=\bigvee_{\{a / x\} \in M}\left(a \odot e_{L^{X}}^{r}(\phi(\{a / x\}), A)\right) \\
&=\bigvee_{\{a / x\} \in M}(a \odot(a \Rightarrow A(x))) \\
& \leq A(x) . \\
& N_{r}(A)(x)=\bigvee_{\{a / x\} \in M}\left(a \odot e_{L^{X}}^{r}(\phi(\{a / x\}), A)\right) \\
&\left.\geq A(x) \odot e_{L^{X}}^{r}(\phi(\{A(x) / x\}), A)\right)=A(x) \odot \top=A(x) .
\end{aligned}
$$

Hence $N_{r}(A)=A$. Similarly, $N_{r}(A)=A$.

(2)

$$
\begin{aligned}
H_{r}(A)(x) & =\bigvee_{\{a / x\} \in M}\left(a \odot \rho_{r}(\phi(\{a / x\}), A)\right) \\
& =\bigvee_{\{a / x\} \in M} \bigvee_{x \in X}(a \odot(a \odot A(x))) \\
& \geq \top \odot(\top \odot A(x))=A(x)
\end{aligned}
$$

Similarly, $A \leq H_{l}(A)$. 
Theorem 14. Let $\phi(\{a / x\})=C$ with $C \in L^{X}$. Then

(1) $N_{r}(A)=e_{L^{X}}^{r}(C, A)$ and $N_{l}(A)=e_{L^{X}}^{l}(C, A)$.

(2) $H_{r}(A)=\rho_{r}(C, A)$ and $H_{l}(A)=\rho_{r}(C, A)$.

Proof. (1)

$$
\begin{aligned}
N_{r}(A)(x) & =\bigvee_{\{a / x\} \in M}\left(a \odot e_{L^{X}}^{r}(\phi(\{a / x\}), A)\right) \\
& =\bigvee_{\{a / x\} \in M}\left(a \odot e_{L^{X}}^{r}(C, A)\right)=e_{L^{X}}^{r}(C, A)
\end{aligned}
$$

(2)

$$
\begin{aligned}
H_{r}(A)(x) & =\bigvee_{\{a / x\} \in M}\left(a \odot \rho_{r}(\phi(\{a / x\}), A)\right) \\
& =\bigvee_{\{a / x\} \in M}\left(a \odot \rho_{r}(C, A)\right)=\rho_{r}(C, A) .
\end{aligned}
$$

Other cases are similarly proved.

Theorem 15. Let $R \in L^{X \times X}$ and define $\phi(\{a / x\})=[x]_{R}$ where $[x]_{R}(y)=$ $R(x, y)$. Then $A(y))$.

(1) $N_{r}(A)(x)=\bigwedge_{y \in X}(R(x, y) \Rightarrow A(y))$ and $N_{l}(A)(x)=\bigwedge_{y \in X}(R(x, y) \rightarrow$

(2) $H_{r}(A)(x)=\bigvee_{y \in X}(R(x, y) \odot A(y))$ and $H_{l}(A)(x)=\bigvee_{y \in X}(A(y) \odot$ $R(x, y))$.

(3) If $R(x, y)=R(y, x)$, then $H_{r}\left(N_{r}(A)\right) \leq A$ and $H_{l}\left(N_{l}(A)\right) \leq A$.

(4) If $R(x, y)=R(y, x)$, then $N_{r}\left(H_{r}(A)\right) \geq A$ and $N_{l}\left(H_{l}(A)\right) \geq A$.

Proof. (1)

$$
\begin{aligned}
N_{r}(A)(x) & =\bigvee_{\{a / x\} \in M}\left(a \odot e_{L^{X}}^{r}(\phi(\{a / x\}), A)\right) \\
& =\bigvee_{\{a / x\} \in M}\left(a \odot e_{L^{X}}^{r}\left([x]_{R}, A\right)\right)=e_{L^{X}}^{r}\left([x]_{R}, A\right) \\
& =\bigwedge_{y \in X}(R(x, y) \Rightarrow A(y)) . \\
N_{l}(A)(x) & =\bigvee_{\{a / x\} \in M}\left(e_{L^{X}}^{l}(\phi(\{a / x\}), A) \odot a\right) \\
& =\bigvee_{\{a / x\} \in M}\left(e_{L^{X}}^{l}\left([x]_{R}, A\right) \odot a\right)=e_{L^{X}}^{l}\left([x]_{R}, A\right) \\
& =\bigwedge_{y \in X}(R(x, y) \rightarrow A(y)) .
\end{aligned}
$$

(2)

$$
\begin{aligned}
H_{r}(A)(x) & =\bigvee_{\{a / x\} \in M}\left(a \odot \rho_{r}(\phi(\{a / x\}), A)\right) \\
& =\bigvee_{\{a / x\} \in M}\left(a \odot \rho_{r}\left([x]_{R}, A\right)\right)=\rho_{r}\left([x]_{R}, A\right) \\
& =\bigvee_{y \in X}(R(x, y) \odot A(y)) .
\end{aligned}
$$




$$
\begin{aligned}
H_{l}(A)(x) & =\bigvee_{\{a / x\} \in M}\left(\rho_{l}(\phi(\{a / x\}), A) \odot a\right) \\
& =\bigvee_{\{a / x\} \in M}\left(\rho_{l}\left([x]_{R}, A\right) \odot a\right)=\rho_{l}\left([x]_{R}, A\right) \\
& =\bigvee_{y \in X}(A(y) \odot R(x, y)) .
\end{aligned}
$$

(3)

$$
\begin{aligned}
H_{r}\left(N_{r}(A)\right)(x) & =\bigvee_{y \in X}\left(R(x, y) \odot \bigwedge_{w \in X}(R(y, w) \Rightarrow A(w))\right) \\
& \leq \bigvee_{y \in X}(R(x, y) \odot(R(y, x) \Rightarrow A(x))) \\
& =\bigvee_{y \in X}(R(x, y) \odot(R(x, y) \Rightarrow A(x))) \\
& \leq A(x) .
\end{aligned}
$$

Similarly, $H_{l}\left(N_{l}(A)\right) \leq A$.

(4)

$$
\begin{aligned}
N_{r}\left(H_{r}(A)\right)(x) & \left.=\bigwedge_{y \in X}\left(R(x, y) \Rightarrow H_{r}(A)(y)\right)\right) \\
& \left.=\bigwedge_{y \in X}\left(R(x, y) \Rightarrow \bigvee_{w \in X}(R(y, w) \odot A(w))\right)\right) \\
& \geq \bigwedge_{y \in X}(R(x, y) \Rightarrow R(y, x) \odot A(x)) \\
& \geq A(x)
\end{aligned}
$$

Similarly, $N_{l}\left(H_{l}(A)\right) \geq A$.

Example 16. Let $K=\left\{(x, y) \in R^{2} \mid x>0\right\}$ be a set and we define an operation $\otimes: K \times K \rightarrow K$ as follows:

$$
\left(x_{1}, y_{1}\right) \otimes\left(x_{2}, y_{2}\right)=\left(x_{1} x_{2}, x_{1} y_{2}+y_{1}\right) .
$$

Then $(K, \otimes)$ is a group with $e=(1,0),(x, y)^{-1}=\left(\frac{1}{x},-\frac{y}{x}\right)$.

We have a positive cone $P=\left\{(a, b) \in R^{2} \mid a=1, b \geq 0\right.$, or $\left.a>1\right\}$ because $P \cap P^{-1}=\{(1,0)\}, P \odot P \subset P,(a, b)^{-1} \odot P \odot(a, b)=P$ and $P \cup P^{-1}=K$. For $\left(x_{1}, y_{1}\right),\left(x_{2}, y_{2}\right) \in K$, we define

$$
\begin{aligned}
\left(x_{1}, y_{1}\right) \leq\left(x_{2}, y_{2}\right) & \Leftrightarrow\left(x_{1}, y_{1}\right)^{-1} \odot\left(x_{2}, y_{2}\right) \in P,\left(x_{2}, y_{2}\right) \odot\left(x_{1}, y_{1}\right)^{-1} \in P \\
& \Leftrightarrow x_{1}<x_{2} \text { or } x_{1}=x_{2}, y_{1} \leq y_{2} .
\end{aligned}
$$

Then $(K, \leq \otimes)$ is a lattice-group.

The structure $\left(L, \odot, \Rightarrow, \rightarrow,\left(\frac{1}{2}, 1\right),(1,0)\right)$ is a generalized residuated lattice with strong negation where $\perp=\left(\frac{1}{2}, 1\right)$ is the least element and $T=(1,0)$ is the greatest element if we define:

$$
\begin{aligned}
& \left(x_{1}, y_{1}\right) \odot\left(x_{2}, y_{2}\right)=\left(x_{1}, y_{1}\right) \otimes\left(x_{2}, y_{2}\right) \vee\left(\frac{1}{2}, 1\right)=\left(x_{1} x_{2}, x_{1} y_{2}+y_{1}\right) \vee\left(\frac{1}{2}, 1\right), \\
& \left(x_{1}, y_{1}\right) \Rightarrow\left(x_{2}, y_{2}\right)=\left(\left(x_{1}, y_{1}\right)^{-1} \otimes\left(x_{2}, y_{2}\right)\right) \wedge(1,0)=\left(\frac{x_{2}}{x_{1}}, \frac{y_{2}-y_{1}}{x_{1}}\right) \wedge(1,0), \\
& \left(x_{1}, y_{1}\right) \rightarrow\left(x_{2}, y_{2}\right)=\left(\left(x_{2}, y_{2}\right) \otimes\left(x_{1}, y_{1}\right)^{-1}\right) \wedge(1,0)=\left(\frac{x_{2}}{x_{1}},-\frac{x_{2} y_{1}}{x_{1}}+y_{2}\right) \wedge(1,0) .
\end{aligned}
$$


We have $(x, y)=(x, y)^{* \circ}=(x, y)^{\circ *}$ from:

$$
\begin{aligned}
& (x, y)^{*}=(x, y) \Rightarrow\left(\frac{1}{2}, 1\right)=\left(\frac{1}{2 x}, \frac{1-y}{x}\right), \\
& (x, y)^{* \circ}=\left(\frac{1}{2 x}, \frac{1-y}{x}\right) \rightarrow\left(\frac{1}{2}, 1\right)=(x, y) .
\end{aligned}
$$

Let $X=\{a, b, c\}$ be a set. Define $\left(R_{1}(a, b)\right),\left(R_{2}(a, b)\right) \in L^{X \times X}$ as

$$
R_{1}=\left(\begin{array}{ccc}
(1,0) & \left(\frac{5}{8}, \frac{5}{2}\right) & \left(\frac{5}{6}, \frac{5}{3}\right) \\
\left(\frac{5}{7}, \frac{30}{7}\right) & (1,0) & \left(\frac{5}{8},-\frac{5}{4}\right) \\
(1,-2) & \left(\frac{5}{7}, \frac{10}{3}\right) & (1,0)
\end{array}\right) R_{2}=\left(\begin{array}{ccc}
(1,0) & \left(\frac{2}{3}, 5\right) & \left(\frac{5}{6}, 1\right) \\
\left(\frac{2}{3}, 5\right) & (1,0) & \left(\frac{6}{7}, 4\right) \\
\left(\frac{5}{6}, 1\right) & \left(\frac{6}{7}, 4\right) & (1,-2)
\end{array}\right)
$$

(1) For $\phi(\{a / x\})=[x]_{R_{1}}$ where $[x]_{R_{1}}(y)=R_{1}(x, y)$ and

$$
\begin{aligned}
A & =\left(\left(\frac{3}{4}, 1\right),\left(\frac{5}{6}, 2\right),\left(\frac{3}{5}, 0\right)\right)^{t}, \\
N_{r}(A)(a) & =\bigvee_{\{k / x\} \in M}\left(k \odot e_{L^{X}}^{r}\left([x]_{R_{1}}, A\right)\right) \\
& =e_{L^{x}}^{r}\left([x]_{R_{1}}, A\right)=\left(\frac{18}{25},-2\right), \\
N_{r}(A)(b) & =\left(\frac{5}{6}, 2\right), N_{r}(A)(c)=\left(\frac{3}{5}, 0\right) \\
H_{r}\left(N_{r}(A)\right)= & \left(\left(\frac{18}{25},-2\right),\left(\frac{5}{6}, 2\right),\left(\frac{18}{25},-4\right)\right)^{t} \not \leq A . \\
N_{l}(A)(a) & =\bigvee_{\{k / x\} \in M}\left(e_{L^{X}}^{l}\left([x]_{R_{1}}, A\right) \odot k\right) \\
& =e_{L^{l}}^{l}\left([x]_{R_{1}}, A\right)=\left(\frac{18}{25},-\frac{6}{5}\right), \\
N_{l}(A)(b) & =\left(\frac{5}{6}, 2\right), \quad N_{l}(A)(c)=\left(\frac{3}{5}, 0\right) \\
H_{l}\left(N_{l}(A)\right)= & \left(\left(\frac{18}{25},-\frac{6}{5}\right),\left(\frac{5}{6}, 2\right),\left(\frac{18}{25},-\frac{86}{25}\right)\right)^{t} \not \leq A . \\
\left.H_{r}(A)\right) & =\left(\left(\frac{3}{4}, 1\right),\left(\frac{5}{6}, 2\right),\left(\frac{3}{4},-\frac{7}{5}\right)\right)^{t}, \\
\left.H_{l}(A)\right) & =\left(\left(\frac{3}{4}, 1\right),\left(\frac{5}{6}, 2\right),\left(\frac{3}{4},-\frac{1}{2}\right)\right)^{t}, \\
N_{r}\left(H_{r}(A)\right) & =H_{r}(A), N_{l}\left(H_{l}(A)\right)=H_{l}(A) .
\end{aligned}
$$

(2) For $\phi(\{a / x\})=[x]_{R_{2}}$ where $[x]_{R_{2}}(y)=R_{2}(x, y)$ and

$$
\begin{gathered}
A=\left(\left(\frac{3}{4}, 1\right),\left(\frac{5}{6}, 2\right),\left(\frac{3}{5}, 0\right)\right)^{t}, \\
N_{r}(A)=\left(\left(\frac{3}{4}, 1\right),\left(\frac{5}{6}, 2\right),\left(\frac{3}{5}, 2\right)\right)^{t},
\end{gathered}
$$




$$
\begin{aligned}
H_{r}\left(N_{r}(A)\right) & =\left(\left(\frac{3}{4}, 1\right),\left(\frac{5}{6}, 2\right),\left(\frac{3}{5}, 0\right)\right)^{t}=A, \\
N_{l}(A) & =\left(\left(\frac{3}{4}, 1\right),\left(\frac{5}{6}, 2\right),\left(\frac{3}{5}, \frac{6}{5}\right)\right)^{t}, \\
H_{l}\left(N_{l}(A)\right) & =\left(\left(\frac{3}{4}, 1\right),\left(\frac{5}{6}, 2\right),\left(\frac{3}{5}, 0\right)\right)^{t}=A, \\
H_{r}(A) & =\left(\left(\frac{3}{4}, 1\right),\left(\frac{5}{6}, 2\right),\left(\frac{3}{5},-2\right)\right)^{t}, \\
N_{r}\left(H_{r}(A)\right) & =\left(\left(\frac{3}{4}, 1\right),\left(\frac{5}{6}, 2\right),\left(\frac{3}{5}, 0\right)\right)^{t}=A, \\
H_{l}(A) & =\left(\left(\frac{3}{4}, 1\right),\left(\frac{5}{6}, 2\right),\left(\frac{3}{5},-\frac{6}{5}\right)\right)^{t}, \\
N_{l}\left(H_{l}(A)\right) & =\left(\left(\frac{3}{4}, 1\right),\left(\frac{5}{6}, 2\right),\left(\frac{3}{5}, 0\right)\right)^{t}=A .
\end{aligned}
$$

Theorem 17. Let $\left(L^{X}, e_{L^{X}}^{r}, e_{L^{X}}^{l}\right)$ be a bi-partial ordered set.

(1) $R=\left\{H_{r}(A) \mid A \in L^{X}\right\}$ is a right join complete lattice.

(2) $S=\left\{H_{l}(A) \mid A \in L^{X}\right\}$ is a left join complete lattice.

(3) $\rho_{r}\left(A, \sqcup_{r} \Phi\right)=\bigvee_{B \in X} \rho_{r}(A, B) \odot \Phi(B)$.

(4) $\rho_{l}\left(A, \sqcup_{l} \Phi\right)=\bigvee_{B \in X} \Phi(B) \odot \rho_{l}(A, B)$.

Proof. (1) For $\Phi \in L^{L^{X}}$,

$$
\begin{aligned}
\sqcup_{r} \Phi(x)= & \bigvee_{A \in L^{X}}\left(H_{r}(A)(x) \odot \Phi\left(H_{r}(A)\right)\right. \\
& =\bigvee_{A \in L^{X}}\left(\bigvee_{\{a / x\} \in M}\left(a \odot \rho_{r}(\phi(\{a / x\}), A)\right) \odot \Phi\left(H_{r}(A)\right)\right. \\
& =\bigvee_{A \in L^{X}}\left(\bigvee_{\{a / x\} \in M}\left(a \odot \bigvee_{y \in X}(\phi(\{a / x\})(y) \odot A(y))\right) \odot \Phi\left(H_{r}(A)\right)\right. \\
& =\bigvee_{A \in L^{X}}\left(\bigvee_{\{a / x\} \in M}\left(a \odot \bigvee_{y \in X}(\phi(\{a / x\})(y) \odot A(y))\right) \odot \Phi\left(H_{r}(A)\right)\right. \\
& =\bigvee_{A \in L^{X}}\left(\bigvee_{\{a / x\} \in M}\left(a \odot \bigvee_{y \in X}\left(\phi(\{a / x\})(y) \odot A(y) \odot \Phi\left(H_{r}(A)\right)\right)\right)\right. \\
& =\bigvee_{A \in L^{X}}\left(\bigvee_{\{a / x\} \in M}\left(a \odot \bigvee_{y \in X}(\phi(\{a / x\})(y) \odot E(y))\right)=H_{r}(E)(x)\right.
\end{aligned}
$$

where $E=A \odot \Phi\left(H_{r}(A)\right)$. Hence $\sqcup_{r} \Phi \in R$.

(2) For $\Phi \in L^{L^{X}}$,

$$
\begin{aligned}
& \sqcup_{l} \Phi(x)=\bigvee_{A \in L^{X}}\left(\Phi\left(H_{l}(A) \odot H_{l}(A)(x)\right)\right. \\
& =\bigvee_{A \in L^{X}}\left(\Phi\left(H_{l}(A) \odot \bigvee_{\{a / x\} \in M}\left(\rho_{l}(\phi(\{a / x\}), A) \odot a\right)\right)\right. \\
& =\bigvee_{A \in L^{X}} \bigvee_{\{a / x\} \in M}\left(\Phi\left(H_{l}\right)(A) \odot \bigvee_{y \in X}(A(y) \odot \phi(\{a / x\})(y)) \odot a\right) \\
& \left.=\bigvee_{\{a / x\} \in M} \bigvee_{y \in X}\left(\bigvee_{A \in L^{X}}\left(\Phi\left(H_{l}\right)(A) \odot A(y)\right) \odot \phi(\{a / x\})(y)\right) \odot a\right) \\
& \left.=\bigvee_{\{a / x\} \in M} \bigvee_{y \in X}(D(y) \odot \phi(\{a / x\})(y)) \odot a\right) \\
& =\bigvee_{\{a / x\} \in M}\left(\rho_{l}(\phi(\{a / x\}), D) \odot a\right) \\
& =H_{l}(D)(x) \text {. }
\end{aligned}
$$


where $D=\Phi\left(H_{l}(A)\right) \odot A$. Hence $\sqcup_{l} \Phi \in S$.

(3)

$$
\begin{aligned}
\rho_{r}\left(A, \sqcup_{r} \Phi\right) & =\bigvee_{x \in X}\left(A(x) \odot \bigvee_{B \in X}(B(x) \odot \Phi(B))\right. \\
& =\bigvee_{B \in X}\left(\bigvee_{x \in X}(A(x) \odot B(x)) \odot \Phi(B)\right) \\
& =\bigvee_{B \in X} \rho_{r}(A, B) \odot \Phi(B)
\end{aligned}
$$

(4)

$$
\begin{aligned}
\rho_{l}\left(A, \sqcup_{l} \Phi\right) & =\bigvee_{x \in X}\left(\sqcup_{l} \Phi(x) \odot A(x)\right) \\
& =\bigvee_{x \in X}\left(\bigvee_{B \in X}(\Phi(B) \odot B(x)) \odot A(x)\right) \\
& =\bigvee_{B \in X}\left(\Phi(B) \odot \bigvee_{x \in X}(B(x) \odot A(x))\right) \\
& =\bigvee_{B \in X} \Phi(B) \odot \rho_{l}(A, B)
\end{aligned}
$$

Theorem 18. (1) If $\{a / x\} \leq \phi(\{a / x\})$, then $N_{r}(A) \leq A \leq H_{r}(A)$ and $N_{l}(A) \leq A \leq H_{l}(A)$.

(2) If $\phi(\{a / x\}) \leq\{a / x\}$, then $N_{r}(A) \leq A \leq H_{r}(A)$ and $N_{l}(A) \leq A \leq$ $H_{l}(A)$.

(3) If $a \odot \phi(\{a / x\})(y) \odot b \leq \phi(\{b / y\})(x)$, then $H_{r}\left(N_{r}(A)\right) \leq A$ for $A \in L^{X}$.

(4) If $b \odot \phi(\{a / x\})(y) \odot a \leq \phi(\{b / y\})(x)$, then $H_{l}\left(N_{l}(A)\right) \leq A$ for $A \in L^{X}$.

(5) If $\phi(\{a / x\})(y) \leq b \odot \phi(\{b / y\})(x)$, then $N_{r}\left(H_{r}(A)\right) \geq A$ for $A \in L^{X}$.

(6) If $\phi(\{a / x\})(y) \leq \phi(\{b / y\})(x) \odot b$, then $N_{l}\left(H_{l}(A)\right) \geq A$ for $A \in L^{X}$.

Proof. (1)

$$
\begin{aligned}
& N_{r}(A)(x)=\bigvee_{\{a / x\} \in M}\left(a \odot e_{L^{X}}^{r}(\phi(\{a / x\}), A)\right) \\
& \leq \bigvee_{\{a / x\} \in M}\left(a \odot e_{L^{X}}^{r}(\{a / x\}, A)\right) \\
&=\bigvee_{\{a / x\} \in M}(a \odot(a \Rightarrow A(x))) \\
& \leq A(x) . \\
& H_{r}(A)(x)=\bigvee_{\{a / x\} \in M^{\prime}}\left(a \odot \rho_{r}(\phi(\{a / x\}), A)\right) \\
&\left.\geq A(x) \odot e_{L^{X}}^{r}(\{A(x) / x\}, A)\right)=A(x) \odot \top=A(x) .
\end{aligned}
$$

Similarly, $N_{l}(A) \leq A \leq H_{l}(A)$.

(2)

$$
\begin{aligned}
H_{r}(A)(x) & =\bigvee_{\{a / x\} \in M}\left(a \odot \rho_{r}(\phi(\{a / x\}), A)\right) \\
& \leq \bigvee_{\{a / x\} \in M}\left(a \odot \rho_{r}(\{a / x\}, A)\right)=\top \odot A(x)=A(x) .
\end{aligned}
$$




$$
\begin{aligned}
N_{r}(A)(x) & =\bigvee_{\{a / x\} \in M}\left(a \odot e_{L^{X}}^{r}(\phi(\{a / x\}), A)\right) \\
& \geq \bigvee_{\{a / x\} \in M}\left(a \odot e_{L^{X}}^{r}(\{a / x\}, A)\right) \\
& =\bigvee_{\{a / x\} \in M}(a \odot(a \Rightarrow A(x))) \\
& \geq A(x) \odot(A(x) \Rightarrow A(x))=A(x)
\end{aligned}
$$

Similarly, $H_{l}(A) \leq A \leq N_{l}(A)$.

$$
\begin{aligned}
& N_{l}(A)(x)=\bigvee_{\{a / x\} \in M}\left(e_{L^{X}}^{l}(\phi(\{a / x\}), A) \odot a\right) \\
& \leq \bigvee_{\{a / x\} \in M}\left(e_{L^{X}}^{l}(\{a / x\}, A) \odot a\right) \\
&=\bigvee_{\{a / x\} \in M}((a \rightarrow A(x)) \odot a) \\
& \leq A(x) . \\
& H_{l}(A)(x)=\bigvee_{\{a / x\} \in M}\left(\rho_{l}(\phi(\{a / x\}), A) \odot a\right) \\
& \geq A(x) \odot\{\top / x\}(x) \odot \top=A(x) \odot \top=A(x) .
\end{aligned}
$$

(3)

$$
\begin{aligned}
& H_{r}\left(N_{r}(A)\right)(x) \\
& =\bigvee_{\{a / x\} \in M}\left(a \odot \rho_{r}\left(\phi(\{a / x\}), N_{r}(A)\right)\right) \\
& =\bigvee_{\{a / x\} \in M}\left(a \odot \bigvee_{y \in X}\left(\phi(\{a / x\})(y) \odot \bigvee_{\{b / y\} \in M}\left(b \odot e_{L_{X}}^{r}(\phi(\{b / y\}), A)\right)\right)\right) \\
& =\bigvee_{\{a / x\} \in M} \bigvee_{y \in X} \bigvee_{\{b / y\} \in M}\left(a \odot\left(\phi(\{a / x\})(y) \odot\left(b \odot e_{L^{X}}^{r}(\phi(\{b / y\}), A)\right)\right)\right) \\
& \leq \bigvee_{\{a / x\} \in M} \bigvee_{y \in X} \bigvee_{\{b / y\} \in M}\left(\phi(\{b / y\})(x) \odot e_{L^{X}}^{r}(\phi(\{b / y\}), A)\right) \\
& \leq \bigvee_{\{a / x\} \in M} \bigvee_{y \in X} \bigvee_{\{b / y\} \in M}(\phi(\{b / y\})(x) \odot(\phi(\{b / y\})(x) \Rightarrow A(x))) \\
& \leq A(x) .
\end{aligned}
$$

$$
\begin{aligned}
& H_{l}\left(N_{l}(A)\right)(x) \\
& =\bigvee_{\{a / x\} \in M}\left(\rho_{l}\left(\phi(\{a / x\}), N_{l}(A)\right) \odot a\right) \\
& \left.=\bigvee_{\{a / x\} \in M}\left(\bigvee_{y \in X}\left(\bigvee_{\{b / y\} \in M}\left(e_{L^{X}}^{r}(\phi(\{b / y\}), A) \odot b\right) \odot \phi(\{a / x\})(y)\right) \odot a\right)\right) \\
& \left.\left.\left.=\bigvee_{\{a / x\} \in M} \bigvee_{y \in X} \bigvee_{\{b / y\} \in M}\left(e_{L^{X}}^{r}(\phi(\{b / y\}), A) \odot b\right) \odot \phi(\{a / x\})(y)\right) \odot a\right)\right) \\
& \leq \bigvee_{\{a / x\} \in M} \bigvee_{y \in X} \bigvee_{\{b / y\} \in M}\left(e_{L^{X}}^{r}(\phi(\{b / y\}), A) \odot \phi(\{a / x\})(y)\right) \\
& \leq A(x) .
\end{aligned}
$$

$N_{r}\left(H_{r}(A)\right)(x)$

$=\bigvee_{\{a / x\} \in M}\left(a \odot e_{L^{X}}^{r}\left(\phi(\{a / x\}), H_{r}(A)\right)\right)$

$=\bigvee_{\{a / x\} \in M}\left(a \odot \bigvee_{y \in X}\left(\phi(\{a / x\})(y) \Rightarrow \bigvee_{\{b / y\} \in M}\left(b \odot \rho_{r}(\phi(\{b / y\}), A)\right)\right)\right)$

$\geq \bigvee_{\{a / x\} \in M} \bigvee_{y \in X} \bigvee_{\{b / y\} \in M}(a \odot(\phi(\{a / x\})(y) \Rightarrow(b \odot \phi(\{b / y\})(x) \odot A(x))))$

$\geq \bigvee_{\{a / x\} \in M} \bigvee_{y \in X} \bigvee_{\{b / y\} \in M}(a \odot(\phi(\{a / x\})(y) \Rightarrow(\phi(\{a / x\})(y) \odot A(x))))$

$\geq \bigvee_{\{a / x\} \in M}(a \odot A(x))=A(x)$. 
(6)

$$
\begin{aligned}
& N_{l}\left(H_{l}(A)\right)(x) \\
& =\bigvee_{\{a / x\} \in M}\left(e_{L^{X}}^{l}\left(\phi(\{a / x\}), H_{l}(A)\right) \odot a\right) \\
& =\bigvee_{\{a / x\} \in M}\left(\bigvee _ { y \in X } \left(\bigvee_{\{b / y\} \in M}((\phi(\{a / x\})(y) \rightarrow A(x) \odot \phi(\{b / y\})(x) \odot b) \odot a)\right.\right. \\
& \geq \bigvee_{\{a / x\} \in M}\left(\bigvee _ { y \in X } \left(\bigvee_{\{b / y\} \in M}((\phi(\{a / x\})(y) \rightarrow A(x) \odot \phi(\{a / x\})(y)) \odot a)\right.\right. \\
& \geq \bigvee_{\{a / x\} \in M}(A(x) \odot a)=A(x) .
\end{aligned}
$$

Theorem 19. (1) If $a \odot \phi(\{a / x\})(y) \odot b \leq \phi(\{b / y\})(x)$ and $\phi(\{a / x\})(y) \leq$ $b \odot \phi(\{b / y\})(x)$, then $H_{r} \circ N_{r}: L^{X} \rightarrow L^{X}$ is a right interior operator and $N_{r} \circ H_{r}: L^{X} \rightarrow L^{X}$ is a right closure operator.

(2) If $b \odot \phi(\{a / x\})(y) \odot a \leq \phi(\{b / y\})(x)$ and $\phi(\{a / x\})(y) \leq \phi(\{b / y\})(x) \odot b$, then $H_{l} \circ N_{l}: L^{X} \rightarrow L^{X}$ is a left interior operator and $N_{l} \circ H_{l}: L^{X} \rightarrow L^{X}$ is a left closure operator.

Proof. (1) (I1) By Theorem 18(1), $H_{r}\left(N_{r}(A)\right) \leq A$.

(I2) By (I1), since $N_{r}$ is an increasing function, $N_{r}\left(H_{r}\left(N_{r}(A)\right)\right) \leq N_{r}(A)$. By Theorem 18(3), $N_{r}\left(H_{r}\left(N_{r}(A)\right)\right) \geq N_{r}(A)$. Hence $N_{r}\left(H_{r}\left(N_{r}(A)\right)\right)=N_{r}(A)$. So, $H_{r}\left(N_{r}\left(H_{r}\left(N_{r}(A)\right)\right)\right)=H_{r}\left(N_{r}(A)\right)$.

$(\mathrm{RI}) e_{L^{X}}^{r}(A, B) \leq e_{L^{X}}^{r}\left(N_{r}(A), N_{r}(B)\right) \leq e_{L^{X}}^{r}\left(H_{r}\left(N_{r}(A)\right), H_{r}\left(N_{r}(A)\right)\right)$.

Thus $H_{r} \circ N_{r}: L^{X} \rightarrow L^{X}$ is a right interior operator.

(C1) By Theorem 18(1), $N_{r}\left(H_{r}(A)\right) \geq A$.

(C2) By (C1), since $H_{r}$ is an increasing function, $H_{r}\left(N_{r}\left(H_{r}(A)\right)\right) \geq H_{r}(A)$. By Theorem 18(1), $H_{r}\left(N_{r}\left(H_{r}(A)\right)\right) \leq H_{r}(A)$. Hence $H_{r}\left(N_{r}\left(H_{r}(A)\right)\right)=H_{r}(A)$. So, $N_{r}\left(H_{r}\left(N_{r}\left(H_{r}(A)\right)\right)\right)=N_{r}\left(H_{r}(A)\right)$.

$(\mathrm{RC}) e_{L^{X}}^{r}(A, B) \leq e_{L^{X}}^{r}\left(H_{r}(A), H_{r}(B)\right) \leq e_{L^{X}}^{r}\left(N_{r}\left(H_{r}(A)\right), N_{r}\left(H_{r}(A)\right)\right)$.

Thus, $N_{r} \circ H_{r}: L^{X} \rightarrow L^{X}$ is a right closure operator.

(2) It is similarly proved as (1).

From Theorems 15 and 19, we obtain the following corollary.

Corollary 20. Let $R \in L^{X \times X}$ and define $\phi(\{a / x\})=[x]_{R}$ where $[x]_{R}(y)=$ $R(x, y)$. If $R(x, y)=R(y, x)$, then $H_{r} \circ N_{r}$ (resp. $\left.H_{l} \circ N_{l}\right)$ is a right (resp. left) interior operator and $N_{r} \circ H_{r}$ (resp. $N_{l} \circ H_{l}$ ) is a right (resp. left) closure operator. 


\section{References}

[1] R. Bělohlávek, Fuzzy closure operator, J. Math. Anal. Appl., 262 (2001), 473-486.

[2] R. Bělohlávek, Lattices of fixed points of Galois connections, Math. Logic Quart., 47 (2001), 111-116.

[3] X. Chen, Q. Li, Construction of rough approximations in setting, Fuzzy Sets and Systems, 158 (2007), 2641-2653.

[4] G. Georgescu, A. Popescue, Non-commutative Galois connections, Soft Computing, 7 (2003), 458-467.

[5] G. Georgescu, A. Popescue, Non-dual fuzzy connections, Arch. Math. Log. 43 (2004), 1009-1039.

[6] U. Höhle, Many Valued Topology and its Applications, Kluwer Academic Publisher, Boston (2001).

[7] U. Höhle, E.P. Klement, Non-Classical Logic and their Applications to Fuzzy Subsets, Kluwer Academic Publisher, Boston (1995).

[8] Z. Pawlak, Rough sets, Int. J. Comput. Inf. Sci., 11 (1982), 341-356.

[9] Z. Pawlak, Rough probability, Bull. Pol. Acad. Sci. Math., 32 (1984), 607615.

[10] W.Z. Wu, W.X. Zhang, Neighborhood operator systems and approximations, Information Sciences, 144 (2002), 201-217.

[11] Y.Y. Yao, Two views of the theory of rough sets in finite universes, Int. J. Approximation Reasoning, 15 (1996), 291-317.

[12] Y.Y. Yao, Constructive and algebraic methods of the theory of rough sets, Information Sciences, 109 (1998), 21-47.

[13] Q.Y. Zhang, W.X. Xie, Fuzzy complete lattices, Fuzzy Sets and Systems, 160 (2009), 2275-2291. 
